\title{
EchoGéo
}

40 | 2017

Ressources urbaines (2)

\section{Trois filiations scientifiques et une piste d'étude}

Conclusion au dossier des ressources urbaines

Monique Bertrand

\section{(2) OpenEdition}

Journals

Electronic version

URL: https://journals.openedition.org/echogeo/14975

DOI: 10.4000/echogeo.14975

ISSN: 1963-1197

Publisher

Pôle de recherche pour l'organisation et la diffusion de l'information géographique (CNRS UMR 8586)

Electronic reference

Monique Bertrand, "Trois filiations scientifiques et une piste d'étude", EchoGéo [Online], 40 | 2017. Online since 26 June 2017, connection on 10 August 2021. URL: http://journals.openedition.org/ echogeo/14975 ; DOI: https://doi.org/10.4000/echogeo.14975

This text was automatically generated on 10 August 2021

EchoGéo est mis à disposition selon les termes de la licence Creative Commons Attribution - Pas d'Utilisation Commerciale - Pas de Modification 4.0 International (CC BY-NC-ND) 


\title{
Trois filiations scientifiques et une piste d'étude
}

\author{
Conclusion au dossier des ressources urbaines
}

\section{Monique Bertrand}

1 Je remercie chaleureusement la rédaction d'ÉchoGéo et les éditrices du généreux dossier consacré aux Ressources urbaines, de m'en laisser quelques pages de conclusion. La proposition est stimulante au regard des dix contributions présentées dans ce double numéro. Mais il est difficile d'ajouter de nouveaux arguments à l'introduction générale, ou une pointe d'originalité à l'égard d'articles rendant compte d'un réel cheminement réflexif: on se trouve avec le tout au cœur d'un champ de recherche cohérent et déjà abouti autour de la notion de ressource, de ses formes urbaines et enjeux cognitifs. Je reviens alors sur quelques héritages scientifiques qui ont pu avoir inspiré de près ou de loin certaines de ces contributions et les échanges qu'elles ont stimulés lors de la journée d'étude « Ressources urbaines et droit à la ville » du Labex Dynamite ${ }^{1}$.

2 «Lire les inégalités socio-spatiales »: l'objectif de connaissance est d'emblée posé à travers la question des ressources urbaines, celle-ci étant posée selon deux agencements : celui des villes et celui de leurs habitants. Mais il ne s'agit pas seulement de qualifier des lieux et des distances, d'illustrer des écarts de consommation et de pratiques, de mesurer les discriminations spatiales mettant les villes en tension à partir d'une définition large de leurs acteurs; il s'agit également de rappeler le bénéfice de l'investigation de terrain, de renvoyer à une méthode de recherche et de mettre en exergue telles ou telles variables d'enquête.

De fait, la confrontation des techniques de production des données participe pour beaucoup à l'intérêt porté aux conditions d'accès à la ville. Au-delà des disparités établies selon les nomenclatures socio-économiques habituelles ou des proxys des niveaux de vie, l'analyse tend vers l'interprétation de ce métabolisme urbain que l'École de Chicago tentait de décrire il y a près d'un siècle. Sur le fond, il s'agit bien de rappeler qu'actifs, résidents, usagers de services urbains, ou simples voisins, fussent-ils de passage et négligés par les politiques publiques, constituent les premiers acteurs de la fabrique des agglomérations, en donnent le pouls intime. Ce faisant, ils sont l'argument 
le plus évident des effets de lieux qu'il convient de décrypter à toutes les échelles d'organisation des espaces urbains.

4 Le potentiel analytique des ressources me semble renvoyer à trois jalons scientifiques. Ils sont ici posés dans des contextes restés longtemps associés, pour les géographes, aux études du développement: ceux de pays puis de villes marqués par de fortes redistributions de population et des transformations territoriales rapides. À leur propos, quelques poncifs sur la planète des bidonvilles et sur la résilience du secteur informel ratent en permanence l'occasion de travailler sur la production mondiale des vulnérabilités et sur leur différenciation locale. Il est donc important de souligner l'apport de ces études "Suds» dans la mesure où elles argumentent des mobilités variées, elles justifient de nouvelles comparaisons internationales entre terrains contrastés au plan économique et peu régulés au plan politique.

\section{L'insertion urbaine des migrants}

5 À bien des égards, le dossier rappelle d'abord le corpus scientifique construit autour de l'arrivée en ville de populations d'origines rurales. Loin de constituer une réminiscence désuète des années 1970, quand les plus grandes villes et les plus fortes croissances urbaines du monde se révèlent au Sud, cette ligne de questionnement des conditions de vie en ville continue de suivre le renouvellement de routes migratoires, notamment internationales, et les flux de migrants les plus récents: certains par passage à la marge, comme à la périphérie de Mexico, d'autres en se greffant sur des filières déjà rodées, comme au centre de la métropole pauliste.

6 L'analyse de ces histoires d'entrée en villes, et d'adaptation aux évolutions que suivent leurs propres marchés du travail et du logement, a suscité en effet une abondante littérature scientifique faisant elle-même écho à d'autres appréciations littéraires et cinématographiques. Elle a surtout posé un important jalon d'étude du fait urbain quand celui se montrait encore minoritaire dans la démographie nationale ou relevait principalement du bilan migratoire. Elle ne tarit pas quand de grandes villes devenues agglomérations imposent l'évidence de flux plus complexes que ceux décrits en termes d'« exode rural », et de lieux d'insertion plus variés que les vieux quartiers centraux et péricentraux. Sans doute aujourd'hui le croît démographique de ces cités repose-t-il de manière plus déterminante sur le bilan naturel construit par les enfants des migrants ruraux, et sur les processus de redistribution interne qui marquent l'étalement urbain. De l'aspiration centripète vers la ville on passe à des dynamiques de reterritorialisation centrifuge, ainsi qu'à des entrées en ville se jouant d'emblée dans les périphéries.

7 Dans cette précédente génération scientifique, le terme d'insertion n'était pas non plus anodin. Même s'il peut paraître aujourd'hui trop fléché au regard de multiples incertitudes, il se démarquait alors, dans le débat français, de celui d'intégration montrant des "étrangers " à la culture urbaine renoncer à leurs déterminations d'origine, comme d'autres migrants devaient s'«assimiler " à marche forcée à la culture nationale dans les termes de politiques migratoires. La notion d'insertion s'est montrée aussitôt plus souple en travaillant ce qui reliait autant que ce qui rompait avec les conditions et caractéristiques de départ. Elle a ainsi imposé ses descripteurs: «trajectoires » biographiques, «filières " de socialisation et de débrouille collective, "réseaux» relationnels susceptibles d'orienter de nouveaux venus vers les cooriginaires déjà installés, puis vers d'autres cercles d'appartenance. Ces mots clefs se 
retrouvent logiquement dans les textes de Laurent Faret et de Sylvain Souchaud. Autour de précarités juridiques ou de nécessités à aborder les réorganisations mondiales de l'activité industrielle, ils traduisent un gradient d'implication sociale dans la migration; ils expriment surtout son épaisseur historique quand le temps du renouvellement de générations s'ajoute dans l'analyse au cycle de vie des individus.

Si elle est restée difficile à traduire notamment en anglais, la problématique de l'insertion rejoint d'autres travaux prolixes sur les chânes de vacance résidentielle, les niches économiques et sur la culture de la pauvreté, pour reprendre les termes d'Oscar Lewis dans le Mexique des années 1960. Songeons par exemple à l'anthropologie des zongo, florissante sur deux décennies d'étude de communautés musulmanes d'Afrique de l'Ouest, et à nombre de monographies sociologiques attachées à telle ou telle population dans ses lieux de prédilection urbaine : tantôt c'est le regroupement des migrants entre eux qui est argumenté ; tantôt le format communautaire semble vite se dissoudre dans des quartiers mixtes et des collectifs de travail hétérogènes. L'observation de la ville s'attache d'abord à la satisfaction de besoins économiques élémentaires mais enchaîne sur la production de sens que signalent ces hommes et ces liens nouveaux, ce qui est alors imputé à une citadinité en gestation. Ni totale déculturation de tribesmen devenant townsmen, pour reprendre les termes discutés en 1961 par Philip Mayer à propos de villes minières d'Afrique australe, ni enfermement dans les attributs d'un sous-prolétariat déraciné : le parcours des migrants, quel que soit leur niveau d'autonomie pris avec le temps, renvoie surtout aux « chances » saisies localement pour entrer et progresser dans l'emploi et dans le marché du logement, qui donnent la première clef de compréhension des ressources urbaines.

C'est sur ce terreau scientifique déjà nourri que s'inscrivent les questionnements géographiques. Pour le spécialiste de la migration rencontrant la réalité métropolitaine en suivant les routes de tel ou tel groupe de ressortissants, fussent-elles contrariées, le dernier arrivé dans une conjoncture de raidissement politique ou de redéploiement économique n'en reste pas moins "entrepreneur» de sa propre mobilité. La plus élémentaire subsistance urbaine devient ressource mobilisée pour le projet migratoire dans son déroulement marqué de verrous externes. Détournée d'une logique de transit, elle est convertie selon les besoins d'un séjour qui se prolonge en ville dans un nouveau champ des possibles: étape improvisée avant rebond vers la destination prévue, déroutement sur un autre trajet, ou reflux à accepter.

10 Le spécialiste de l'urbain semble quant à lui dans une sorte de bifurcation analytique face aux lieux que les flux produisent en ville. Pour certaines études, force est d'admettre que les «migrants» qualifiés ou revendiqués d'abord comme tels finiront par se considérer avant tout comme des citadins. Une nouvelle enquête les reconnaîtra alors en charge d'un projet plus urbain que migratoire, soucieux d'optimiser leurs positions en ville plutôt que d'identifier de nouveaux horizons de vie, "experts » de connaissances dédiées à ces fins, de parades aux risques que présentent les grandes agglomérations, ou encore engagées sur une voie d'émancipation féminine. C'est bien ce qui se passe dans les favelas de Rio abordées par Rafael Soares Gonçalves: la comparaison s'affranchit progressivement de l'opposition des migrants et des nonmigrants et de la différenciation des migrants internes et des migrants internationaux. Ou bien elle maintient des spécificités suscitées par telle ou telle origine régionale, comme le fait Sylvain Souchaud depuis des ateliers de confection, pour mieux différencier, selon d'autres critères, les migrants redevables du même point de départ : 
primo-arrivants versus nouveaux venus, patrons des ateliers versus main-d'œuvre ouvrière, propriétaires versus hébergés sur place. Mais pour d'autres études, il s'agit de suivre avant tout les lieux, espaces d'arrivée et de redistribution dans la ville, non plus tel ou tel groupe de population. Comprendre la structuration d'une agglomération selon différents apports générationnels impose ainsi de réviser régulièrement les typologies de l'habitat, comme le font Vincent Berdoulay et Thi Thu Trang Ngo sur le terrain périurbain de Hô Chi Minh Ville, et pour le moins d'en comparer les quartiers selon des compositions démographiques toujours hétérogènes. Si les variables d'origine restent bien décisives, elles entrecroisent leurs effets de manière plus complexe avec le temps.

11 La première option du suivi de migrants est prise par les textes complémentaires d'Antonine Ribardière et d'Aurélia Michel depuis l'État mexicain de Oaxaca jusque dans le municipe industriel de Naucalpan à Mexico. On saisit ici tout l'intérêt d'ajouter au cadrage statistique du dernier recensement les données d'enquête qualitative ciblant des femmes, actives et mères de famille, en particulier. Des caractéristiques des lieux d'origine on passe aux trajectoires individuelles et familiales, déterminantes puis discriminantes pour comprendre comment l'emploi de service domestique ou le logement locatif sont ciblés, comment l'environnement urbain fini par être jaugé selon des connaissances pratiques et des jeux de difficultés qui ne peuvent apparaître que relatifs à ce type de migrant(e)s.

12 La seconde option du suivi de quartier ressort mieux du texte de Jean-François Valette qui «boucle » en quelque sorte la place donnée à la capitale mexicaine dans ce double numéro: non plus comme scène migratoire, terminus ou de présence discrète, mais comme complexité métropolitaine. Sous le singulier donné au " quartier » dans le titre, le gigantisme démographique et l'épaisseur pluri-générationnelle de Mexico se dévoilent dans les colonies populaires de périphérie urbaine. Celles-ci passent d'un statut de front pionnier valorisant des atouts avant tout locaux pour l'accès à la propriété du logement, puis sa formalisation, à celui de bassin de vie multipliant les services de proximité mais banalisant aussi la mobilité sortante d'une population variée vers d'autres secteurs urbains. C'est finalement l'intensité et l'orientation de ces connexions dans l'agglomération qui différencient les colonies les unes des autres. Mais sur le fond, la question n'est plus celle de l'insertion des migrants et devient celle de l'intégration de quartiers au reste de la ville.

\section{Le paradigme longitudinal}

13 Du cheminement migratoire aux recompositions résidentielles, de l'histoire de vie à la l'histoire de ville, on retrouve ce glissement analytique dans une deuxième source d'argumentation de la notion de ressource urbaine: il s'agit désormais d'apprécier concrètement l'effet du temps sur les pratiques citadines, d'en mesurer la portée à différentes échelles de durée en s'inspirant de méthodes longitudinales. La filiation n'est pas seulement méthodologique; elle est aussi épistémologique, "paradigmatique " comme l'énonçait Daniel Courgeau en traçant un espace de vie depuis des lieux à la fois successifs et simultanés, et en montrant comme les expériences individuelles mesurées par la démographie faisaient résonner les expériences sociales abordées dans d'autres disciplines. 
14 Notons qu'aucun texte du dossier ne découle d'un tel parti pris d'investigation : ni pour rendre compte de données produites rétrospectivement en suivant un calendrier biographique complet, ni pour progresser dans l'observation en continu d'une même cohorte de population. Seule l'exploitation de plusieurs sources statistiques successives permet à Jean-François Valette de suivre le même échantillon, avec le recul de 2007 pour la plus ancienne, mais alors à condition de prendre les colonies populaires pour des "individus" enrichis en population et en tissu socio-économique au fur et à mesure de leur histoire urbaine.

15 Plusieurs textes rappellent cependant l'apport d'approches longitudinales de la migration et de l'insertion urbaine, en particulier à partir de ce qui s'organise en ville depuis l'enjeu du logement : la mobilité résidentielle, la demande de nouveaux services urbains, des négociations entre conjoints en matière d'emploi, les solidarités familiales mobilisées pour la garde d'enfants. Tout cela a déjà bien souligné le rôle d'autres déplacements, métropolitains, les efforts déployés par les citadins dans leurs navettes domicile-travail et pour aborder d'autres ressources de la vie urbaine, les risques de déclassement encourus quand les cartes de l'emploi et de la résidence sont «mal appariées ». La référence à cette strate d'étude devient explicite : soit pour expliquer comment certains formats d'enquêtes se sont imposés sur le terrain, soit pour justifier l'analyse de « choix » et « d'arbitrages ».

16 Une première source d'inspiration consiste à décrire la densité et la variété des expériences citadines sur des bases plus sûres que celles de données déjà agrégées: sources trop anciennes, mal conçues ou biaisées pour répondre aux questions posées. Il s'agit alors d'enquêter un " ancrage en ville » en prenant le recul qui convient à toute tranche de vie : en remontant s'il le faut à "avant la migration », en poursuivant par " une fois le logement stabilisé » pour montrer comment une ressource maitrisée en appelle une autre. Dans tous les cas la compréhension de la ville se dilate dans le temps en même temps qu'elle se diffracte selon différentes échelles spatiales d'observation. Telles sont les conditions pour interroger la variabilité des comportements individuels. À un niveau élémentaire de comparaison, c'est déjà ce que proposait Oscar Lewis en reconstituant une biographie de la vie locative dans le centre de Mexico. Dans ce périmètre familial d'investigation, l'expérience urbaine Sanchez se montrait différente pour le primo-arrivant et pour sa descendance, pour les filles et les garçons d'une même fratrie, pour celui des enfants qui s'éloignait momentanément de la capitale et pour d'autres échouant à s'extraire de la condition ouvrière.

Quelques années plus tard, Philippe Haeringer $(1972,1983)$ fondait en terre ivoirienne " un modèle d'interview biographique et sa transcription synoptique » en comparant quelques "périples » migratoires des années 1970, et en s'attachant par exemple à «trois citadins jour après jour ... dans la compétition abidjanaise ». Fondée sur l'application en série d'un même canevas d'information, le chercheur délaissait la logique familiale d'investigation pour dérouler en parallèle trois récits venant de migrants étrangers les uns aux autres: celui de la formation, du travail et des alternances entre emploi et inactivité; celui de l'émancipation individuelle et de la constitution d'une nouvelle entité domestique ; celui enfin de la résidence tracée dans un enchaînement d'étapes migratoires et de lieux de vie urbains. Suivant ce fil chronologique, des solidarités d'origine, d'étapes et de destination faisaient ressortir la cohérence des parcours, des logiques propres à chacun des migrants plutôt que des liens forts déjà établis entre eux à l'enquête. 
18 À l'appui de plus gros échantillons de population, les enquêtes quantitatives menées enfin sur questionnaires démographiques fermés, sur le modèle «triple B » ou par comparaison de plusieurs cohortes, ont formalisé le traitement des calendriers biographiques. Dans les études consacrées par exemple à "Trois générations de citadins au Sahel », autour de Philippe Antoine, dans les regards croisés animés par le Groupe de Réflexion sur Approche Biographique de l'INED, des "événements», des " séjours", des "états" de vie sont analysés pour eux-mêmes et comme jalons de «carrières » et de « cursus ». C'est bien l'ensemble, le stock de réponses collectées aux deux échelles d'un individu et d'un large échantillon, qui font ressortir les trois versants - matrimonial, professionnel et résidentiel - d'une même vie plus ou moins fléchée, chaotique face aux incertitudes, ou ouverte sur de véritables marges de manœuvre.

19 L'enjeu est donc de modéliser chacune de ces perspectives d'accomplissement social dans son déroulement propre, dans ses rapports avec les autres, ainsi qu'avec le contexte décrit, lui-même en transformation. La démarche fait sens aussi dans les villes abordées dans ce dossier qui se trouvent fortement recomposées en quelques décennies. Elle permet de mieux qualifier les orientations prises par chacun comme étant ascendantes ou régressives, de situer les parcours entre promotions et blocages. Les interactions mesurées entre divers calendriers renvoient aux questions posées sur un mode qualitatif sur de plus petits échantillons : quels sont, ainsi, sur les autres axes de la vie urbaine, les effets de transitions sociales normées comme le passage à l'âge adulte? Les suites d'accidents de parcours comme une instabilité conjugale ou un chômage prolongé ? Ou encore les répercussions d'un changement de conjoncture, comme en ont signalé les programmes d'ajustement structurel pour les économies en développement? Dans le cas de Santiago du Chili abordé par Claudia Arellano Yévenes et Fernando Saavedra Peláez, c'est l'introduction du Transantiago qui modifie le contexte technique et gestionnaire local et conduit les autres modes de déplacement à se repositionner dans l'agglomération. Dans celui du Cap décrit par Solène Baffi, de même, la fin de l'apartheid et l'avènement de gouvernements métropolitains suscitent la promotion des pratiques combinées de transport qui amène à relire la portée de la ségrégation urbaine.

20 Quels que soient ses formats méthodologiques, l'approche longitudinale valorise en effet la notion de choix. Celui-ci se révèle par un changement d'état, soit un résultat plutôt qu'une intention. Plutôt que d'être déduit d'une rationalité donnée d'avance à chaque individu, selon de purs calculs de coûts dans l'économie libérale, le choix s'entend comme une expérience en cours et en interaction avec d'autres, ce qui précisément permet de dépasser l'individualisme méthodologique. C'est l'option qui s'impose dans un champ des possibles construit par un collectif de pratiques, comme la résultante de contraintes et d'anticipations entre deux moments de vie, dans un entourage donné et selon un contexte lui aussi caractérisé.

21 Dans ce continuum d'analyse de la vie urbaine, la mobilité prend tout son sens : non seulement pour faire suite à la migration, pour décrire de nouveaux déménagements, une stabilisation ou des captures résidentielles, mais aussi pour relier les lieux de vie dans la ville. De moyen pour satisfaire des besoins incompressibles et accéder à de nouvelles ressources, le déplacement urbain devient lui-même ressource, enjeu de stratégies et de rêves d'amélioration. Dans cette perspective, il en va des usages du transport comme de recours migratoires et d'options résidentielles : tous questionnent 
une prise de décision qui peut s'envisager par défaut - le "je n'ai pas le choix » de certaines personnes enquêtées -, mais relève la plupart du temps d'arbitrages posés sur la durée à l'égard d'un stock de possibilités construit collectivement. Le cas de Bouaké en donne une illustration dans les termes de l'informalité et du risque accidentel mesuré par Kouakou Konan. Dans les quelques textes consacrés aux déplacements des citadins, la focale s'est en effet déplacée du temps biographique et de tranches de vie à des séquences plus courtes dans les rythmes du quotidien et de la semaine. Sur le fond, c'est bien la même sémantique : des " opportunités » à double tranchant - un avantage immédiat et des externalités négatives -, des alternatives sur une échelle de temps donné, des "portefeuilles» d'options et des « répertoires " d'informations localisées. Des images et des calculs de coût-distance-temps sont ainsi mobilisés à propos de différents lieux de la ville, révélant d'autres différences: de moyens financiers, de capital social et de genre.

22 L'approche longitudinale complète donc l'analyse des marchés du travail et du logement avec celle de la mobilité urbaine, devenue elle aussi une affaire marchande. Par-delà les infrastructures physiques et les types de gestion, les inégalités de recours éclairent d'autres variables de la vie en ville, confirmant la compréhension organique et relationnelle des ressources urbaines. Dans un cas c'est la distance des périphéries urbaines aux centralités économiques qui se trouve au cœur de l'analyse : le transport à deux roues à Santiago conduit à différencier des cyclistes «nouveaux » et " classiques ", les premiers gagnants et les seconds manquant d'alternative; de même, l'intégration des banlieues noires au redéploiement territorial de la ville post-apartheid reste critique tant que le système de transport public est radial. Dans d'autre cas, illustrés par Aurélia Michel ou Vincent Berdoulay et Thi Thu Tran Ngo, la répartition des rôles masculins et féminins dans la famille et la gestion des temps domestiques font mieux ressortir les mécanismes de prise de décision.

23 Au final, l'analyse géographique a déplacé ses objectifs de comparaison des quartiers: aux critères topologiques et de composition démographique qui les faisaient déjà ressortir comme attractifs pour certaines populations ou pour d'autres comme tremplins vers d'autres horizons de ressources, s'ajoutent leurs qualités relationnelles et l'intensité des mobilités les articulant à l'ensemble urbain, les reliant à d'autres lieux évalués comme forts ou faibles.

\section{Capabilités, accessibilité, politiques urbaines}

Dans la vague néolibérale des années 1980, l'effondrement de l'État providence et les politiques d'austérité budgétaire qui sont mises en œuvre un peu partout dans le monde ont enfin de profondes répercussions sur les villes : leurs services publics, leurs aménités sont réévalués selon des logiques de récupérations des coûts et de compétitivité marchande. L'assainissement, la collecte des déchets, l'adduction d'eau, l'énergie montent en puissance comme problèmes d'iniquité à traiter d'autant qu'ils interfèrent avec ceux de l'accès aux soins et à l'éducation, qui entrent désormais dans la définition de l'indice de développement humain. Un pan important des ressources urbaines prend donc le relais analytique de l'emploi et du logement, terrain de mesure habituel de l'attractivité des villes, pour rendre compte de comment s'entretiennent, s'exacerbent ou se déplacent les inégalités socio-économiques; comment l'accroissement général des besoins de consommation installent de nouvelles 
vulnérabilités et trappes de pauvreté dans les villes. Celles des Suds sont vues moins au prisme de leurs migrants qu'à celui de leurs pauvres. Ils sont alors théorisés comme « effets sociaux » d'une décennie perdue pour le développement, celle des crises de la dette publique, puis comme révélateurs de nouvelles ressources adaptatives, celles de l'informel. Les favelas décrites par Rafael Soares Gonçalves en attestent en manifestant un double attachement : aux sociétés d'approvisionnement en eau et aux ressources du voisinage, fussent-elles sous le sceau d'une précarité foncière historique ; bien des pans économiques de cette informalité subsistent d'ailleurs même lorsque leurs habitants se rallient à des logiques de planification.

Les politiques de lutte contre la pauvreté et d'investissement pro-poor qui suivront ce tournant majeur ont d'importantes conséquences méthodologiques. Puisqu'il s'agit d'évaluer concrètement les effets d'improvement de ces politiques, la mesure des subsistances $^{2}$ fait l'objet d'un retournement de méthode dans de grandes enquêtes commandées pour suivre les niveaux de vie et leurs écarts : plutôt que de peser le "panier de la ménagère » selon les revenus des ménages, ce sont les consommations qui sont mesurées. Elles fondent alors l'idée de recours et de capacités, et justifient l'orientation libérale de ces politiques quand il s'agit de « remettre les pauvres dans le marché ». De même la demande de services est re-questionnée sous le double angle du ability and willingness to pay dans de nombreuses enquêtes soutenues par la Banque mondiale.

On mesure ici l'influence de modèles de décision basés sur les agents, et plus largement des libertés de choix abordées comme capabilites par Amartya Sen suivant une définition large des ressources, monétaires, sociales, et spatiales, des individus. À sa manière, le présent dossier participe de ce tournant en mettant en exergue le terme de l'accessibilité : non seulement parce que la desserte des services et l'impact territorial des infrastructures font l'objet d'une attention ancienne en géographie, mais aussi parce que les déplacements conduisant aux équipements et aux réseaux physiques, quels que soient les caractères intrinsèques de ceux-ci, sont désormais compris comme une compétence sociale et une capacité à tirer parti d'informations urbaines. Seront ainsi distingués des migrants démunis en capital scolaire plutôt que d'autres, des primo-accédants à la propriété plutôt que d'autres résidents, des femmes plutôt que des hommes, etc.

Dans cette optique, l'approche scientifique des inégalités d'accès tient de plus en plus compte de leur approche politique. Elle conduit à envisager un spectre élargi de pratiques, incluant la sécurisation foncière et la préservation de ressources environnementales; elle s'attache à des orientations souvent versatiles ou des formes de zapping d'un recours à l'autre: public ou privé, formel ou informel, licite ou clandestin, etc. L'analyse intègre le traitement réglementaire, les jeux de pouvoir et de négociation dont ces facilités urbaines font l'objet, au fil de politiques publiques, de projets ciblés et d'incitations à meilleure gouvernance. Elle voit également des normes internationales $\mathrm{d}^{\prime}$ ' inclusion pour tous » faire leur chemin vers toutes les villes. Au lieu de seulement compléter, mettre à niveau et réformer l'offre d'équipements, il s'agit de mettre tous les utilisateurs potentiels sur un pied d'égalité, d'en désenclaver donc certains en particulier, d'en accroître les « libertés positives » en considérant la valeur qu'ils donnent aux biens et aux services demandés.

Suivant cette dernière filiation, la notion de ressource peut alors s'enrichir de nouvelles observations et typologies en montrant comment migrants et habitants cherchent ou 
non, et surtout de manière inégale, à faire levier dans ces politiques, à en élargir les concertations, ou à peser d'une manière ou d'une autre sur la gestion des villes. Des revendications de droits ou de légitimité, des mobilisations sociales, des négociations par la petite porte, des protestations épisodiques ou des résistances frontales, des " choix » entre réagir après-coup et anticiper... tout cela signe aussi le manque de visibilité des uns et l'empowerment des autres. Une démarche de comparaison s'impose de nouveau face à de véritables répertoires d'action, un éventail d'options et de (non) recours impliquant des formes d'inhibition, des doubles jeux politiques, des implications intermittentes, des plaidoyers en réseau... C'était d'ailleurs la piste ouverte par la journée d'étude d'octobre 2015, dont le titre lancé sur les ressources se complétait en « droit à la ville».

29 Par-delà la formule incantatoire retenue de l'ouvrage d'Henri Lefebvre dans bien des scènes internationales, le pari d'analyse des ressources politiques mobilisées par les citadins à l'égard des transformations de leur propre cadre de vie mérite attention. S'il est relevé dans certaines villes du Nord autour des notions de participation et de citoyenneté, à l'appui d'enquêtes de sociologie politique, c'est moins le cas à la lecture de ce dossier : les auteurs s'y concentrent sur la dimension spatiale des inégalités sans relever systématiquement les stratégies de conquête de nouveaux atouts pour les apaiser. Seuls les textes de Laurent Faret et de Rafael Soares Gonçalves se réfèrent au champ associatif et à des organisations de la société civile mobilisés pour soutenir les droits de migrants clandestins, qui sont alors orientés vers une aide juridique ou d'hébergement, ou pour réévaluer les besoins des favelas au regard des quartiers légaux voisins.

C'est donc sur le terrain du "politique par le bas " que l'on peut suggérer quelques approfondissements de la notion de ressource urbaine, notion que de croissantes revendications de droits ne manqueront pas de convier au débat de la subjectivation politique. Et d'abord sur quelles ressources se concentrer? À quels droits donner une priorité pratique : à un accès équitable à l'eau ? À des loyers contrôlés ? À la survie de vendeurs ambulants? À la fin d'un monopole de transport ? À des soins gratuits? À une carte scolaire ouverte? De nombreuses traductions locales font d'ores et déjà jouer les alternatives : droits sociaux ou droits humains? Droits sectoriels ou universels? Les marges de manœuvre se montrent surtout de plus en plus variées sur nos terrains d'enquête. Mais alors quelles durées de mobilisation? Quelles histoires de leadership ? Quelles trajectoires d'engagement, s'il y a lieu? Sur ce terrain d'analyse aussi pourraient se retravailler les méthodologies et les concepts laissés par de précédentes écoles scientifiques.

31 C'est bien le mérite de ce dossier que de suivre une voie d'enrichissement de la notion de ressources en lui ouvrant la perspective de nouveaux chantiers d'enquête. Certains textes ont valorisé le point de vue de migrants, d'autres celui de citadines ; certains se sont attachés à des quartiers typés, d'autres se sont focalisés sur des connexions dans la ville. Dans tous les cas, il s'agissait bien de partir de pratiques habitantes, toujours finement localisées ici, pour dépasser une vision de fabrique de la ville et de sa gouvernance limitée à quelques "parties prenantes ». De plus humbles acteurs et des pratiques ordinaires sont ressortis de nombreuses mesures de position économique, mais aussi de l'appréciation de sociabilités, de normes culturelles, de stratégies cognitives, et finalement d'un sens politique. Reconnaître l'universalité de ces processus dans trois continents, argumenter la singularité des réponses apportées 
localement, et souvent dans la précarité, à de larges faisceaux de contraintes individuelles, sociales et contextuelles : le défi pour ce double numéro est bien relevé.

\section{BIBLIOGRAPHY}

Antoine Ph. (ed.), Ouédraogo D. (ed.), Piché V. (ed.), Diop A.B. (préf.), 1998. Trois générations de citadins au Sahel : trente ans d'histoire sociale à Dakar et à Bamako. Paris, L'Harmattan, $283 \mathrm{p}$.

Courgeau D., 1975. Le concept de migration. In Institut National de la Statistique et de la Démographie (ed.), Actes du quatrième colloque de démographie africaine : migrations, état-civil, recensements administratifs, p. 27-37.

Courgeau D., 2002. Évolution ou révolutions dans la pensée démographique ?. Mathématiques et sciences humaines, $\mathrm{n}^{\circ} 160$, p. 49-76.

GRAB, 2009. Biographies d'enquêtes. Bilan de 14 collectes biographiques. Paris, INED Méthodes et savoirs, $\mathrm{n}^{\circ}$ 3, IRD, RSEH, $340 \mathrm{p}$.

Haeringer Ph, 1972. Méthodes de recherche sur les migrations africaines : un modèle d'interview biographique et sa transcription synoptique. Cahiers de l'ORSTOM, série Sciences humaines, vol. IX-4.

Haeringer Ph, 1983. Trois citadins jour après jour : un Ebrié, un Baoulé et un Mossi dans la compétition abidjanaise. Cahiers de l'ORSTOM, série Sciences humaines, vol 19-4.

Lefebvre H., 1967. Le droit à la ville. L'Homme et la société, nº 6, p. 29-35.

Lewis O., 1978 (trad. rééd.). Les enfants de Sanchez. Autobiographie d'une famille mexicaine. Paris, Gallimard, 644 p.

Mayer Ph., 1961. Townsmen or Tribesmen: Conservatism and the Process of Urbanization in a South African City (Contributions by Iona Mayer). Capetown, South African, Oxford University Press Rhodes University, $306 \mathrm{p}$.

Sen A., 2012 (trad. réed.). Éthique et économie. Paris, Puf, 372 p.

\section{NOTES}

1. Journée d'étude du 15 octobre 2015 organisée à l'Université de Paris Diderot par Aurélia Michel et Antonine Ribardière, Laboratoire d'Excellence Dynamique Territoriales et Spatiales : Groupe de travail 2.4. « Produire la ville "ordinaire" ».

2. De nombreux projets de développement sont reformulés autour de fonds livelihoods. 
INDEX

Subjects: Sur le Champ - Sur le Terrain

\section{AUTHOR}

\section{MONIQUE BERTRAND}

Monique Bertrand, monique.bertrand@ird.fr, est directrice de recherche à l'Institut de recherche pour le développement, membre du CESSMA, Centre d'études en sciences sociales sur les mondes africains, américains et asiatiques - UMR 245 IRD. 